\title{
circEPS15 Overexpression in Hepatocellular Carcinoma Modulates Tumor Invasion and Migration
}

\section{Maolin Tian}

Peking University Third Hospital

\section{Gang Li}

Peking University Third Hospital

Bin Jiang

Peking University Third Hospital

\section{Sadula Abuduhaibaier}

Peking University Third Hospital

\section{Dianrong Xiu}

Peking University Third Hospital

\section{Chunhui Yuan}

Peking University Third Hospital

\section{Yuntao Bing ( $\boldsymbol{\sim}$ bingyuntao@bjmu.edu.cn )}

Peking University Third Hospital https://orcid.org/0000-0002-0187-133X

\section{Primary research}

Keywords: circular RNA, hepatocellular carcinoma, EPS15, microarray, ceRNA

Posted Date: August 11th, 2020

DOl: https://doi.org/10.21203/rs.3.rs-54670/v1

License: (c) (i) This work is licensed under a Creative Commons Attribution 4.0 International License. Read Full License 


\section{Abstract}

Background: Hepatocellular carcinoma (HCC) is one of the leading causes of cancer-related deaths worldwide. Recent evidence indicates that circular RNAs (circRNAs) play important roles in tissue development, gene regulation, and carcinogenesis. However, whether circRNAs are involved in HCC progression and encode functional proteins remains largely unknown.

Methods: Circular RNA microarrays were performed using three pathologically diagnosed HCC samples and their paired adjacent normal liver tissues. Cell invasion, migration, cell cycle, and apoptosis after circRNA overexpression were measured using a transwell culture system, a wound healing assay, and flow cytometry . Full-length, mutated, and truncated sequences of circEPS15 with a FLAG tag were inserted inside a circular expression vector. Western blotting was used to confirm circEPS15 expression and the requirement of internal ribosomal entry site (IRES) elements within the circRNA. The miRNA and mRNA expression profiles were obtained by analyzing data retrieved from The Cancer Genome Atlas (TCGA) database. We then constructed a ceRNA network of mRNAs, miRNAs, and circEPS15. Using tissue samples from own patients, we also verified certain analytical results with quantitative real-time PCR (qRT-PCR).

Results: The expression of circEPS15 was downregulated in HCC tissues, and the survival curves showed that low circEPS15 levels were associated with poor overall survival in HCC patients. Overexpression of circEPS15 suppressed tumor invasion and migration by inhibiting the TJP1/CDH2/VIM signaling pathway and retarded cell cycle progression, but it had no effect on cell apoptosis. ceRNA analysis and qRT-PCR showed that there might be a circRNA (circEPS15)-miRNA (miR-24-3p)-mRNA (CIDEA) network in HCC. The spanning junction open reading frame in circEPS15 driven by IRES encoded a novel protein.

Conclusions: Endogenous circEPS15 plays a novel role in repressing HCC through the ceRNA network and encoding a functional protein.

\section{Introduction}

Hepatocellular carcinoma (HCC) is one of the most common malignant tumors observed in the clinic; it ranks fifth in the incidence of malignant tumors and the third leading cause of mortality[1]. Invasion, metastasis, and postoperative recurrence are the main causes of death in patients with HCC. Epithelial mesenchymal transition (EMT) is the process of transforming epithelial cells into mesenchymal cells with specific physiological or pathological conditional phenotypes[2]. In recent years, EMT has been found to be closely related to the recurrence and metastasis of HCC and has become a hot topic in cancer research. Revealing the molecular regulatory mechanism underlying EMT and the relationship between EMT and malignant tumors would assist in the search for new molecular targets [2,3].

Circular RNA (circRNA) is different from traditional linear RNA. It has a 5 '- 3' polar covalent closed loop structure and is relatively stable, abundant, and conserved in the eukaryotic transcriptome. Recent studies have shown that the expression of circRNAs is tissue- and cell-specific [4-6]. An increasing amount of 
evidence shows that circRNA is associated with the occurrence, development, prognosis, and prognosis of cancer [7-9]; however, the role and mechanism of circRNAs in HCC has not been elucidated. The hepatitis B virus is prevalent in China, and the incidence of primary HCC is also higher than that in developed countries such as Europe and the United States[10]. Therefore, it is urgent and important to understand the role of circRNA in the occurrence and development of HCC and provide an effective target for HCC diagnosis and treatment.

$\mathrm{HCC}$ is a complex molecular process driven by gene mutations accompanied by epigenetic modifications [11]. At present, there is no effective diagnosis or treatment strategy, and thus, it is urgent to find a potential effective diagnostic marker and therapeutic target. In recent years, an increasing number of noncoding RNAs, including small interfering RNA (siRNA) and long non-coding RNA (IncRNA), have been found to be scrambled in patients with HCC that may have potential clinical value [12]. However, the expression and function of circRNAs in HCC have not been elucidated.

Studies have shown that circRNA imbalance is associated with many human diseases and may be related to the occurrence and progression of systemic lupus erythematosus, diabetes, and Alzheimer's disease [13-15]. Other studies have shown that circRNAs are involved in the occurrence and development of tumors, including colon, gastric, and cervical cancers [16-19]. Although the study of circRNAs has received increased attention in the past five years, the biological functions and mechanisms of many circRNAs have not been elucidated, and it is not known whether these circRNAs are transcribed.

The molecular mechanism of circRNA biological function mainly includes miRNA absorption through the sponge effect, which affects the biological function of the miRNA [20]. In the second pathway, circRNA is a biological regulator that binds to proteins [21]. At present, some studies have reported that circRNA can translate functional proteins to play a role in glioma tumorigenesis [22]. However, whether circRNA plays a biological role in HCC and its underlying mechanism has not been elucidated.

In the past decade, a large number of studies have shown that miRNAs in circulation can be used as potential biomarkers for pancreatic cancer, Barrett's esophagus and esophageal adenocarcinoma [23, 24]. However, although a large number of clinical studies have been conducted to HBV-related HCC and HCVrelated $\mathrm{HCC}[25,26]$, the instability of miRNAs and difficulty in reproducing results under different experimental conditions make miRNAs a poor biomarker for clinical practice. In contrast, circRNA is more stable because of its closed-loop structure and tolerance to a large number of RNA enzymes present in the environment. Moreover, because of its tissue-specific expression [27], circRNA could be an ideal biomarker for predicting the occurrence, progression, and prognosis of disease.

With further research of the mechanism of circRNA, it may have a potential therapeutic use in the future. Additionally, because of its stability, circRNA can be used as a continuous cell regulator. For example, a specific circRNA can be synthesized by genetic engineering to play a specific biological role through the binding of specific miRNA, competing for binding ribosomal protein, or translating a small polypeptide. $A$ study showed that an overexpressed synthesized circRNA could absorb miRNA-122 through sponge-like interactions; thus, affecting the replication cycle of the hepatitis $\mathrm{C}$ virus (HCV) through the inhibition of 
HCV protein synthesis [28]. Therefore, synthetic circRNA has broad application prospects in the field of molecular medicine.

In the present study, we investigated the tumor-suppressive role of circRNA-100226 in HCC using paired human $\mathrm{HCC}$ and para-cancerous tissues. Using the human reference genome, we further estimated that the circRNA located at chr1:51868106-51874004 is derived from EPS15 (epidermal growth factor receptor pathway substrate 15), which is located on chromosome $1 \mathrm{p} 32.3$. Thus, we termed circRNA100226 "circESP15". We also overexpressed circEPS15 to further explore the functions and mechanisms of tumor growth.

\section{Materials And Methods}

\section{Patients and specimens}

The study was approved by the Ethic Committee of Third Hospital, Peking University. Written consent was obtained from all patients prior to surgery. A total of $71 \mathrm{HCC}$ tissues and matched adjacent nontumor tissues were obtained from patients who underwent surgery in the Third Hospital, Peking University. None of the patients had received chemotherapy, radiotherapy, or other anticancer therapy before surgery. The patients were histologically confirmed by experienced pathologists. Paired tissue specimens (tumor and adjacent normal tissues) were collected from the patients, immediately frozen in liquid nitrogen and then stored at $-80^{\circ} \mathrm{C}$ until use. Clinical information and follow-up data were collected and listed in Table 1.

\section{CircRNAs microarray hybridization and data analysis}

Total RNAs were digested with RNase R (20U/ $\mu$ l, Epicentre, USA) to remove linear RNAs and enrich circular RNAs. The enriched circular RNAs were amplified and transcribed into fluorescent cRNA utilizing a random priming method (Super RNA Labelling Kit; Arraystar, Rockville, MD, USA). The labelled cRNAs were hybridized onto the Human circRNA Array $(8 \times 15 \mathrm{~K}$, Arraystar). The slides were incubated for $17 \mathrm{~h}$ at $65^{\circ} \mathrm{C}$ in a hybridization oven (Agilent, Santa Clara, CA, USA). CircRNAs differentially expressed with statistical significance between $\mathrm{HCC}$ and paired normal tissues (fold-change $(\mathrm{FC}) \geq 2$ and $\mathrm{p} \leq 0.05$ ) were identified through Volcano Plot filtering. Hierarchical clustering was performed to show the distinguishable expression pattern of circRNAs among samples.

\section{Cell Culture}

The human HCC cell lines (BEL-7402, BEL-7404, Huh7, Hep3B and HepG2) and normal hepatocyte cell $\mathrm{HL}-7702$ were purchased from the Shanghai Institute of Cell Biology with a certificate of authenticity for each cell line. The samples were maintained in DMEM/RPMI1640 (Gibco BRL, Grand Island, NY, USA) supplemented with $10 \%$ heat-inactivated fetal calf serum, $2 \mathrm{mM} \mathrm{L-glutamine,} \mathrm{and} 100 \mathrm{U} / \mathrm{mL}$ penicillinstreptomycin mixture (Gibco BRL, Grand Island, NY, USA) at $37^{\circ} \mathrm{C}$ in $5 \% \mathrm{CO} 2$. After undergoing cell culture in our laboratory for several months, all cell lines used were evaluated for genetic identification 
prior to the experiments. Based on the appraisal reports provided by CoBioer Biosciences Co., Ltd., no mutations and contaminations were found in our cell lines.

\section{Quantitative reverse transcription polymerase chain reaction (qRT-PCR)}

All primers used for qRT-PCR were designed in primer 5.0 software (Premier, Canada) and synthesized by Tsing Ke Biotech (Chengdu, China). The complementary DNAs (cDNAs) for circRNA, mRNA, or miRNA measurement were synthesized using random, oligo(dT)18 or stem-loop primers, respectively, using RevertAid First Strand cDNA Synthesis Kit (Thermo Fisher Scientific, Waltham, MA, USA). qRT-PCR was performed in triplicate using a Maxima SYBR Green qPCR Master Mix (Thermo Fisher Scientific) on the CFX connect real-time system (Bio-Rad, Hercules, CA, USA). Glyceraldehyde-3-phosphate dehydrogenase (GAPDH) was employed as the intrinsic control for circRNA and mRNA, and U6 was used as the endogenous control for miRNA. Relative expression level of each gene was calculated using the $2-\Delta \Delta C t$ method.

\section{5. circEPS15-overexpressing vector construction and transduction}

The circEPS15-overexpressing plasmid synthesized and cloned into the adenoviral vector GV138 (Genechem) with a Flag-tag. Full-length circEPS15 and its serial deletion performed using X-tremeGENE siRNA transfection reagent (Roche, Mannheim, Germany). For western blot analysis, cell proteins were prepared $72 \mathrm{~h}$ post transfection.

\section{Wound-Healing Assay}

The cells were seeded in 6-well plates and transfected following the manufacturer's instructions. When the cell confluence reached approximately $80 \%$ at $48 \mathrm{~h}$ posttransfection, cells were serum-starved for 12 $\mathrm{h}$ and then scratched within the confluent cell layer by using the fine end of a 100-IL pipette tip. Wound healing was observed at different time points within the scrape line. At this point, representative scrape lines were photographed by using an inverted Leica phase-contrast microscope (Leica DFC $300 \mathrm{FX}$ ) under $20 \mathrm{X}$ objective lens every $24 \mathrm{~h}$. Image Processing and Analysis in Java was used to measure the woundhealing assays. Duplicate wells for each condition were examined, and each experiment was repeated three times.

\section{Trans-well Assay}

Cell invasion assay was performed in a trans-well chamber (24-well type, $8 \mathrm{~mm}$ pore size, Corning, NY, USA). BD Matrigel Basement Membrane Matrix was assessed according to the manufacturer's recommended protocol. Then, $0.5 \mathrm{~mL}$ of serum-free medium was placed in the upper chamber; DMEM with $10 \%$ FBS was added to the bottom chambers. Equal numbers (1X105) of cells were plated in the upper chambers of the quadruplicate wells and incubated at $37^{\circ} \mathrm{C}$ for $72 \mathrm{~h}$. Cells were then fixed with paraformaldehyde and stained with crystal violet, to visualize the nuclei. The results of three independent experiments were averaged. Image Processing and Analysis in Java was used to measure the trans-well assay. 


\section{Cell cycle and apoptosis assay}

To assess the cell cycle and apoptosis, $3 \times 105$ treated cells were seeded into 6-well plates and cultured for $48 \mathrm{~h}$ at $37^{\circ} \mathrm{C}$. The cells for cell cycle analysis were digested using trypsin (Hyclone), washed twice with phosphate-buffered saline (PBS), and fixed in $70 \%$ ethanol overnight at $4^{\circ} \mathrm{C}$. The cells were centrifuged at $500 \mathrm{~g}$ for $5 \mathrm{~min}$, washed twice with cold PBS, and centrifuged. After treating with RNase $\mathrm{A}(0.1 \mathrm{mg} / \mathrm{ml})$ and propidium iodide $\left(\mathrm{Pl}, 0.05 \mathrm{mg} / \mathrm{ml}\right.$ ) purchased from $4 \mathrm{~A}$ Biotech (Beijing, China) for $30 \mathrm{~min}$ at $37^{\circ} \mathrm{C}$, cell cycle analysis was performed through fluorescence-activated cell sorting flow cytometry (Beckman Coulter, Palo Alto, CA, USA). For the analysis of apoptosis, cells were trypsinised followed by two PBS washing steps. The cells were stained using the Annexin V/PI detection kit (4A Biotech, Beijing, China) for 5 min at room temperature. The apoptotic cells were measured using flow cytometry (Beckman Coulter). All experiments were repeated at least three time.

\section{Western blot analysis}

For the detection of protein, cytoplasm and nuclear protein extracts were prepared from cells. The protein concentration of each sample was determined using a NanodropTM spectrophotometer (Thermo Scientific). Protein (100ug) from each sample was examined bySDS-PAGE(4\% stacking and $10 \%$ separating gels) and then transferred overnight on to PVDF membranes(Millipore). The membranes were immunoblotted with the following: poly clonal anti-human TJP1 antibody (1:200, Abgent); CDH2 antibody (1:1000, Abcam); VIM antibody (1:1000, Cell Signaling Technology); EPS15 antibody (1:500, Abgent); GAPDH antibody (1:1000, Santa Cruz Biotechnology); Flag antibody (1:1000, Cell Signaling Technology) overnight. The blots were then incubated with peroxidase-conjugated goat anti-rabbit antibody or goatanti-mouse antibody (1:4000, Millipore) for $1 \mathrm{~h}$. The PVDF membranes were subsequently subjected to immunoblotting analysis using an ECL immunoblotting kit according to the manufacturer's recommended protocol (Beyotime Institute of Biotechnology, China).

\section{IRES prediction}

The IRESfinder[29] was used to identify the IRES sequence of circRNA. Each sequence of circRNA was evaluated by sliding window approach with window size of $174 \mathrm{nt}$ and step size of $50 \mathrm{nt}$. The region with the highest score was considered to be the IRES sequence of circRNA.

\section{Statistical analyses}

All statistical analyses were performed using SPSS version 21.0 (IBM SPSS Inc., Chicago, IL, USA) and Prism version 5.0 (GraphPad Software, LaJolla, CA, USA) softwares. Categorical variables were expressed as a count or percentage and tested using $\chi 2$ or Fisher's exact test, as appropriate. Continuous data are reported as mean \pm standard deviation (SD) and compared using Student's $t$ test, the one-way analysis of variance (ANOVA) test or Mann-Whitney test as appropriate. Correlations were calculated using Pearson's correlation analysis. The cut-off value used to stratify patients into high and low expression groups was the median expression of target genes. Survival curves were plotted using the Kaplan-Meier method and 
compared using the log-rank test. All tests were 2-sided, and $\mathrm{P}<0.05$ was considered statistically significant.

\section{Results}

\section{circRNA expression profiles in HCC}

To explore the potential involvement of circRNAs in HCC, we performed circRNA microarray analyses on two pairs of HCC tissues $(T)$ and matched para-carcinoma normal tissues $(C)$. We found 608 circRNAs that were upregulated in HCC tissue with fold change greater than 2, $\mathrm{P}<0.05$, while 737 circRNAs were downregulated using the same cutoff parameters (shown in Fig. 1a). Using hierarchical clustering, we identified the top five upregulated and downregulated circRNAs in HCC tissues (shown in Fig. 1b), and the variation in circRNA expression was summarized in the scatter and volcano plots (shown in Fig. 1c-d). To verify the expression of these top five upregulated and downregulated circRNAs in 32 randomly selected hepatoma adjacent and their tissues, we used q-RT-PCR and junction primers. circEPS15 and circRNA103595 were the top down- and up-regulated circRNAs in HCC tissues, respectively, which was consistent with our microarray analysis (shown in Fig. 1e).

\section{Correlation between circEPS15 expression and HCC clinical characteristics}

To further investigate the role of circEPS15 in HCC, the relationship between circEPS15 expression in HCC tissues and the clinical pathological characteristics of HCC patients was analyzed. As shown in Table 1, low expression of circEPS15 in HCC tissues was significantly correlated with larger tumor size $(p=0.01)$, but not with poor tumor differentiation $(P=0.01)$. However, other characteristics of $\mathrm{HCC}$, including the AFP level, tumor number, BCLC stage, and vascular invasion, were also not associated with circEPS15 expression. These data provide evidence of the importance of circEPS15 in the growth and tumorigenesis of HCC.

\section{Prognostic and diagnostic value of circEPS15 and circRNA-103595}

Survival information from 71 patients was available at the end of the clinical follow-up, from which Receiver Operating Characteristic (ROC) analysis was performed to determine whether circEPS15 and circRNA-103595 had diagnostic significance. Results showed that the Area Under Curve (AUC) of circEPS15 and circRNA-103595 was 0.735 and 0.627 , respectively, which confirmed that circEPS15 was diagnostically significant for HCC (shown in Fig. 2a-b). Kaplan-Meier analysis of the correlation between circEPS15 and patient survival showed that low circEPS15 level was significantly associated with a poor overall survival (shown in Fig. 2c).

\section{Effects of circEPS15 on HCC tumor cell proliferation and cell cycle progression}

Based on these results, we wanted to further study the specific function of circEPS15 in HCC formation and development. We confirmed that the tumor cell lines HL-7402 and HL-7404 had low expression of circEPS15 compared to normal cell lines HL-7702 and L02. We constructed an adenovirus vector to 
overexpress circEPS15 in HL-7402 and HL-7404 cell lines. After transfection, the expression of circEPS15 increased in HL-7402 cells but not in HL-7404 cells, indicating successful transfection and overexpression of circEPS15 in HL-7402 (shown in Fig. 3a); therefore, we used HL-7402 for further study. We investigated the effect of circEPS15 overexpression on the cell cycle by measuring the percentage of HCC cells in different cell cycle stages (e.g. G1/G0, S, and G2/M) and found that circEPS15 overexpression increased the percentage of cells in G2/M compared to the vector control (shown in Fig. $3 \mathrm{~b})$. In addition, we detected the apoptosis of transfected cells compared with the negative control, and the results showed no significant difference (shown in Fig. 3c). These results indicated that circEPS15 elicited cell cycle arrest but did not affect apoptosis.

\section{Overexpression of circEPS15 suppressed HCC cell migration and invasion by inhibiting the TJP1/CDH2/VIM signaling pathway}

Because circEPS15 suppressed tumor growth, we subsequently investigated the effect of circEPS15 on HCC function, including migration and invasion. Wound healing and transwell assays indicated that circEPS15 significantly increased HCC cell migration and invasion compared to negative control (NC) (shown in Fig. 3d-e). To investigate the molecular mechanism underlying the specific function of circEPS15 in HCC, we tested several proteins involved in the signaling pathways that affect cell migration and invasion using western blotting. Overexpression of circEPS15 reduced TJP1 and VIM protein expression, but induced the expression of $\mathrm{CDH} 2$ (shown in Fig. 3f). These results indicated that circEPS15 inhibited HCC cell migration and invasion by affecting the proteins involved in the TJP1/CDH2/VIM signaling pathway.

\section{Prediction of circRNA-miRNA interactions and competing endogenous RNA (ceRNA) regulatory network analyses}

CircRNAs act as microRNA sponges associated with related miRNAs, and together they made up the circRNA-miRNA axis involved in disease pathogenesis. To determine the function of circEPS15, its target miRNAs were predicted with informatics analysis using TargetScan (http://www.targetscan.org/vert_72/) and miRanda (http://www.microrna.org/microrna/home.do). The top five miRNAs related to each validated circRNA and the predicted circEPS15 interaction sites are shown in Fig. 4. To further elucidate the underlying mechanism of circEPS15, a circRNA-miRNA-mRNA regulatory network was constructed using Cytoscape (shown in Fig. 5a). The network suggested that circRNAs could indirectly regulate miRNA target genes by competitively binding to miRNAs. In order to further clarify that circEPS15 can regulate its target gene, 11 target genes were tested by qRT-PCR, which showed that there was a positive correlation between cricEPS15 and CEDIA (shown in Fig. 5B and 5C). These results suggest that circEPS15 may regulate CEDIA through a ceRNA regulatory network, which affects the process of epithelial mesenchymal transition.

\section{Evaluation of circEPS15 protein-coding ability}


CircEPS15's sequence was matched in circRNADb; therefore, we next analyzed the putative open reading frame (ORF) in circEPS15 (shown in Fig. 6a). There was a potential spanning junction ORF in circEPS15 that could encode a 150-amino acid protein. Conservation analysis showed that this ORF was highly conserved among different species, suggesting that this ORF was translatable. To test the putative ORF transcription ability, a wild type (ATG) or mutation type (AAG) circEPS15 was cloned and inserted into the GV486 vector (shown in Fig. 6b). To test the putative IRES activity in circEPS15, a full-length or truncated putative circEPS15 IRES was also cloned into the GV486 vector. To add a FLAG tag to the gene of interest, we moved the junction to the stop codon, with side flanking sequences (circEPS15-FLAG) (shown in Fig. 6b). We transfected these plasmids into 293T cells and detected their potential translated products. As shown in Fig. 6c, the FLAG antibody only detected a $25 \mathrm{kDa}$ protein in wild-type circEPS15FLAG (OE5) and circEPS15 (ins_FLAG)-IRES1del (OE4) transfected cells but not in the mutated (AAG), IRES2 truncated, or IRES1 and IRES2 truncated circEPS15-FLAG transfected cells. The scores for the IRES prediction in these five sequences were consistent with the western blotting results, wherein OE4 and OE5 had the highest scores (0.75) by IRESFinder (shown in Table 2). These results indicated that circEPS15 could encode a protein, the ORF was translatable, and ATG and IRES2 were essential for the 5 '-cap independent translation of circEPS15.

\section{Discussion}

Since the demonstration of the stable expression of numerous circRNAs in eukaryocytes [30], there has been increasing interest in characterizing the role of circRNAs in various diseases. circRNAs are novel RNA molecules with different biological functions and pathological implications [30-32], including the "miRNA sponge" [20,30,33]; however, the more general roles of circRNAs remain elusive [34, 35]. Interestingly, artificial circRNA was shown to be translatable in eukaryotic cells [36] and current evidence has also shown that other types of non-coding RNAs can initiate protein synthesis, raising the question of whether endogenous circRNAs can encode proteins in mammalian cells [37-39]. The expression profile of circEPS15 in HCC and its underlying mechanism remain unknown, and in the present study, we used microarray and qRT-PCR analyses combined with in vivo experiments to reveal how the downregulation of circEPS15 in HCC tissues is associated with HCC prognosis.

The downregulation of circEPS15 in HCC tissues was significantly associated with tumor differentiation and intravascular tumor thrombus, indicating that circEPS15 functions as a tumor suppressor and might be important for the epithelial mesenchymal transition of HCC. This hypothesis was validated in vitro and in vivo. Moreover, we demonstrated that circEPS15 could regulate CEDIA through a ceRNA regulatory network and have protein-coding ability.

EPS15 is transcribed from Ch38.p13, which encodes a protein that is a part of the EGFR pathway [40]. The protein is present in clathrin-coated pits and involved in receptor-mediated endocytosis of epidermal growth factor (EGF) [41]. Moreover, alternative splicing results in multiple transcript variants encoding distinct isoforms [42]. 
Apart from influencing cell migration and cancer-related signaling, EPS15 is also correlated with poor prognosis in several tumor types, including breast cancer, esophageal squamous cell carcinoma, and glioma [43-45]. A previous study showed that EDH2 (Eps15 homology domain-containing 2) inhibited migration and invasion of hepatocellular carcinoma, probably by interacting with E-cadherin [46]. However, the potential mechanism of EPS15 in tumor suppression remains unclear. Consistent with previous studies, EPS15 mRNA was significantly downregulated in HCC tissues compared with the matched non-tumorous tissues, and mRNA-EPS15 expression levels in HCC tissues were positively correlated with circESP15. These observations indicate that the expression of circEPS15 in HCC may be mainly regulated by the amplification of the host gene. Recently, Bai et al. [47] and He et al. [48] reported that circFBLIM1 and circGRFA1 can regulate corresponding linear FBLIM1 and GRFA1 mRNA transcript expression through a ceRNA mechanism.

In this study, by matching the RNA deep sequencing data with circRNADb, we found that circEPS15 could encode a protein in vitro. Given the critical role of the EPS15 gene in tumorigenesis, we hypothesized that the identical functions of EPS15 isoforms, as well as shorter and more stable circEPS15 translated proteins, may serve as critical regulators to control cellular proliferation. This study was limited to cultured cells; therefore, the clinical significance of circEPS15 needs to be addressed in a larger clinical cohort. Furthermore, although the tumor-suppressive role was definitive and we provide clear evidence that circRNA encodes a functional protein in vivo, the clinical application of circEPS15 must still be validated. circRNAs have been reported to be important regulators during critical biological processes; however, no translatable circRNAs or their products have been reported in HCC. Our findings expand the current understanding of circRNAs and imply that the coding potential of circRNAs is largely underestimated. More specifically, our evidence of circEPS15 expression and activity suggests that circEPS15 and its translated protein may play a role in HCC progression and patient clinical prognosis.

\section{Declarations}

\section{Statement of Ethics}

This study was approved by Peking University Third Hospital Medical Science Research Ethics Committee. Written informed consent was obtained from all patients who provided HCC and precancerous samples and the principles of the Declaration of Helsinki were followed.

\section{Conflict of Interest Statement}

The authors have no conflict of interest.

\section{Funding}

The research was supported by grants from the National Natural Science Foundation of China (No. 81702855).

\section{Availability of data and materials}


The analyzed data sets generated during the present study are available from the corresponding author on reasonable request.

\section{Acknowledgement}

Not applicable.

\section{Corresponding Author}

Correspondence to Yuntao Bing, Chunhui Yuan and Dianrong Xiu

\section{Author information}

Maolin Tian, Gang Li and Bin Jiang contribute equally to this work as co-first authors.

\section{Author contributions}

B.Y.T. and Y.C.H. performed the described studies, analyzed data, and prepared the manuscript. T.M.L, L.G. and J.B. provided clinical and experiments suggestions. T.M.L., L.G., J.B. and A.S. provided materials and assisted in experiments. X.D.R. and Y.C.H. advised on study design and prepared the manuscript.

\section{References}

1. Olofson, A.M., et al., Steatohepatitic Variant of Hepatocellular Carcinoma: A Focused Review. Gastroenterology Res, 2018. 11(6): p. 391-396.

2. Tam, W.L. and R.A. Weinberg, The epigenetics of epithelial-mesenchymal plasticity in cancer. Nat Med, 2013. 19(11): p. 1438-49.

3. Xie, G. and A.M. Diehl, Evidence for and against epithelial-to-mesenchymal transition in the liver. Am J Physiol Gastrointest Liver Physiol, 2013. 305(12): p. G881-90.

4. Jeck, W.R., et al., Circular RNAs are abundant, conserved, and associated with ALU repeats. RNA, 2013. 19(2): p. 141-57.

5. Lu, T., et al., Transcriptome-wide investigation of circular RNAs in rice. RNA, 2015. 21(12): p. 2076-87.

6. Salzman, J., et al., Circular RNAs are the predominant transcript isoform from hundreds of human genes in diverse cell types. PLoS One, 2012. 7(2): p. e30733.

7. Chen, X., S. Fan, and E. Song, Noncoding RNAs: New Players in Cancers. Adv Exp Med Biol, 2016. 927: p. 1-47.

8. Li, F., et al., Circular RNA ITCH has inhibitory effect on ESCC by suppressing the Wnt/beta-catenin pathway. Oncotarget, 2015. 6(8): p. 6001-13.

9. Starke, S., et al., Exon circularization requires canonical splice signals. Cell Rep, 2015. 10(1): p. 10311. 
10. Yu, R., R. Fan, and J. Hou, Chronic hepatitis B virus infection: epidemiology, prevention, and treatment in China. Front Med, 2014. 8(2): p. 135-44.

11. Bing, Y., et al., Glucocorticoid-induced S-adenosylmethionine enhances the interferon signaling pathway by restoring STAT1 protein methylation in hepatitis B virus-infected cells. J Biol Chem, 2014. 289(47): p. 32639-55.

12. He, Y., et al., Long noncoding RNAs: Novel insights into hepatocelluar carcinoma. Cancer Lett, 2014. 344(1): p. 20-27.

13. Li, C., et al., Correct microarray analysis approaches in 'Hsa-circRNA11783-2 in peripheral blood is correlated with coronary artery disease and type 2 diabetes mellitus'. Diab Vasc Dis Res, 2018. 15(1): p. 92-93.

14. Li, H., et al., Comprehensive circular RNA profiles in plasma reveals that circular RNAs can be used as novel biomarkers for systemic lupus erythematosus. Clin Chim Acta, 2018. 480: p. 17-25.

15. Zhao, Y., et al., Deficiency in the Ubiquitin Conjugating Enzyme UBE2A in Alzheimer's Disease (AD) is Linked to Deficits in a Natural Circular miRNA-7 Sponge (circRNA; ciRS-7). Genes (Basel), 2016. 7(12).

16. Gao, Y.L., et al., Circular RNA expression profiles reveal that hsa_circ_0018289 is up-regulated in cervical cancer and promotes the tumorigenesis. Oncotarget, 2017. 8(49): p. 86625-86633.

17. Hsiao, K.Y., et al., Noncoding Effects of Circular RNA CCDC66 Promote Colon Cancer Growth and Metastasis. Cancer Res, 2017. 77(9): p. 2339-2350.

18. Huang, M., et al., Circular RNA hsa_circ_0000745 may serve as a diagnostic marker for gastric cancer. World J Gastroenterol, 2017. 23(34): p. 6330-6338.

19. Weng, W., et al., Circular RNA ciRS-7-A Promising Prognostic Biomarker and a Potential Therapeutic Target in Colorectal Cancer. Clin Cancer Res, 2017. 23(14): p. 3918-3928.

20. Zheng, Q., et al., Circular RNA profiling reveals an abundant circHIPK3 that regulates cell growth by sponging multiple miRNAs. Nat Commun, 2016. 7: p. 11215.

21. Zeng, Y., et al., A Circular RNA Binds To and Activates AKT Phosphorylation and Nuclear Localization Reducing Apoptosis and Enhancing Cardiac Repair. Theranostics, 2017. 7(16): p. 3842-3855.

22. Yang, Y., et al., Novel Role of FBXW7 Circular RNA in Repressing Glioma Tumorigenesis. J Natl Cancer Inst, 2018. 110(3).

23. Gablo, N.A., et al., Cell-free microRNAs as Non-invasive Diagnostic and Prognostic Bio- markers in Pancreatic Cancer. Curr Genomics, 2019. 20(8): p. 569-580.

24. Lv, J., et al., Circulating exosomal miRNAs as potential biomarkers for Barrett's esophagus and esophageal adenocarcinoma. World J Gastroenterol, 2020. 26(22): p. 2889-2901.

25. Li, J., et al., Serum microRNA expression profiling identifies serum biomarkers for HCV-related hepatocellular carcinoma. Cancer Biomark, 2019. 26(4): p. 501-512.

26. Morishita, A., et al., Role of microRNA-210-3p in hepatitis B virus-related hepatocellular carcinoma. Am J Physiol Gastrointest Liver Physiol, 2020. 318(3): p. G401-G409. 
27. Melo, S.A., et al., Glypican-1 identifies cancer exosomes and detects early pancreatic cancer. Nature, 2015. 523(7559): p. 177-82.

28. Jost, l., et al., Functional sequestration of microRNA-122 from Hepatitis C Virus by circular RNA sponges. RNA Biol, 2018. 15(8): p. 1032-1039.

29. Zhao, J., et al., IRESfinder: Identifying RNA internal ribosome entry site in eukaryotic cell using framed k-mer features. J Genet Genomics, 2018. 45(7): p. 403-406.

30. Memczak, S., et al., Circular RNAs are a large class of animal RNAs with regulatory potency. Nature, 2013. 495(7441): p. 333-8.

31. Rybak-Wolf, A., et al., Circular RNAs in the Mammalian Brain Are Highly Abundant, Conserved, and Dynamically Expressed. Mol Cell, 2015. 58(5): p. 870-85.

32. You, X., et al., Neural circular RNAs are derived from synaptic genes and regulated by development and plasticity. Nat Neurosci, 2015. 18(4): p. 603-610.

33. Hansen, T.B., et al., Natural RNA circles function as efficient microRNA sponges. Nature, 2013. 495(7441): p. 384-8.

34. Guo, J.U., et al., Expanded identification and characterization of mammalian circular RNAs. Genome Biol, 2014. 15(7): p. 409.

35. Jeck, W.R. and N.E. Sharpless, Detecting and characterizing circular RNAs. Nat Biotechnol, 2014. 32(5): p. 453-61.

36. Chen, C.Y. and P. Sarnow, Initiation of protein synthesis by the eukaryotic translational apparatus on circular RNAs. Science, 1995. 268(5209): p. 415-7.

37. Lauressergues, D., et al., Primary transcripts of microRNAs encode regulatory peptidesPrimary transcripts of microRNAs encode regulatory peptides. Nature, 2015. 520(7545): p. 90-U205.

38. Magny, E.G., et al., Conserved Regulation of Cardiac Calcium Uptake by Peptides Encoded in Small Open Reading Frames. Science, 2013. 341(6150): p. 1116-1120.

39. Nelson, B.R., et al., A peptide encoded by a transcript annotated as long noncoding RNA enhances SERCA activity in muscle. Science, 2016. 351(6270): p. 271-275.

40. Niehof, M. and J. Borlak, EPS15R, TASP1, and PRPF3 are novel disease candidate genes targeted by HNF4alpha splice variants in hepatocellular carcinomas. Gastroenterology, 2008. 134(4): p. 1191202.

41. Morgan, J.R., et al., Eps15 homology domain-NPF motif interactions regulate clathrin coat assembly during synaptic vesicle recycling. J Biol Chem, 2003. 278(35): p. 33583-92.

42. Kieken, F., et al., Mechanism for the selective interaction of C-terminal Eps15 homology domain proteins with specific Asn-Pro-Phe-containing partners. J Biol Chem, 2010. 285(12): p. 8687-94.

43. Li, M., et al., Effects of EHD2 interference on migration of esophageal squamous cell carcinoma. Medical Oncology, 2013. 30(1).

44. Shi, Y.H., et al., Decreased expression and prognostic role of EHD2 in human breast carcinoma: correlation with E-cadherin. Journal of Molecular Histology, 2015. 46(2): p. 221-231. 
45. Smith, J.S., et al., A transcript map of the chromosome 19q-arm glioma tumor suppressor region. Genomics, 2000. 64(1): p. 44-50.

46. Liu, J., et al., Decreased Expression of EHD2 Promotes Tumor Metastasis and Indicates Poor Prognosis in Hepatocellular Carcinoma. Dig Dis Sci, 2016. 61(9): p. 2554-67.

47. Bai, N., et al., circFBLIM1 act as a ceRNA to promote hepatocellular cancer progression by sponging miR-346. Journal of Experimental \& Clinical Cancer Research, 2018. 37.

48. He, R.F., et al., circGFRA1 and GFRA1 act as ceRNAs in triple negative breast cancer by regulating miR-34a. Journal of Experimental \& Clinical Cancer Research, 2017. 36.

\section{Figures}


Fig.1
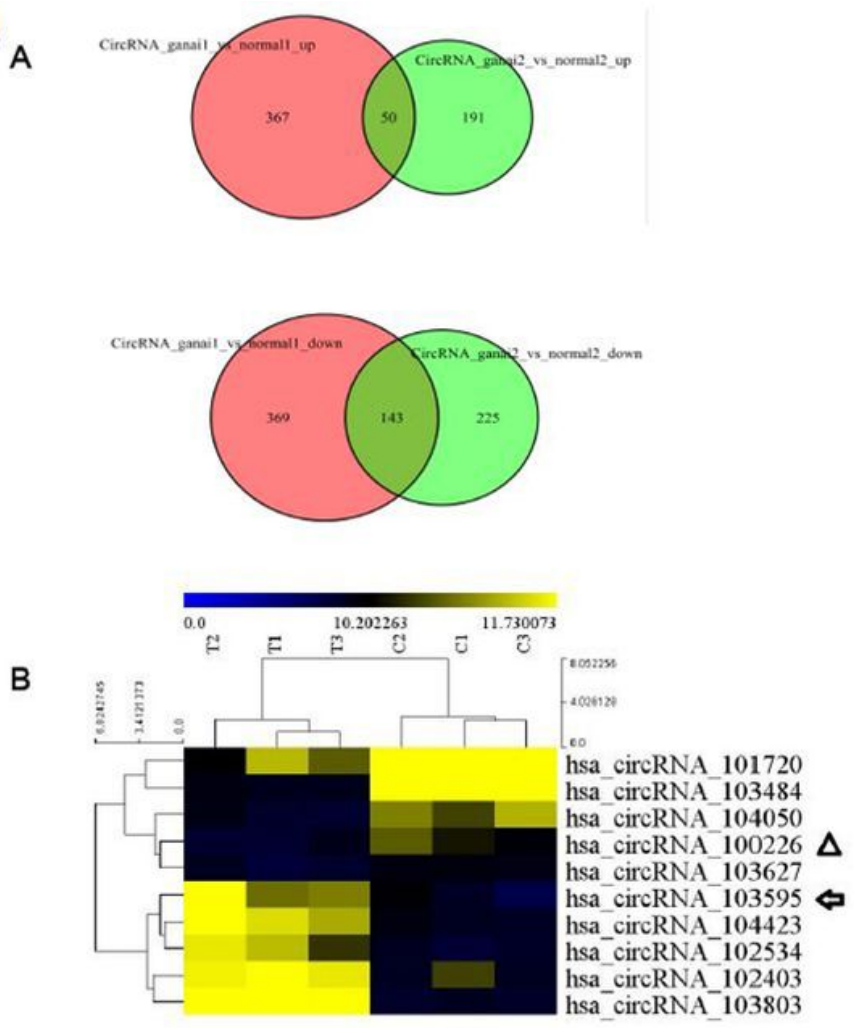

E

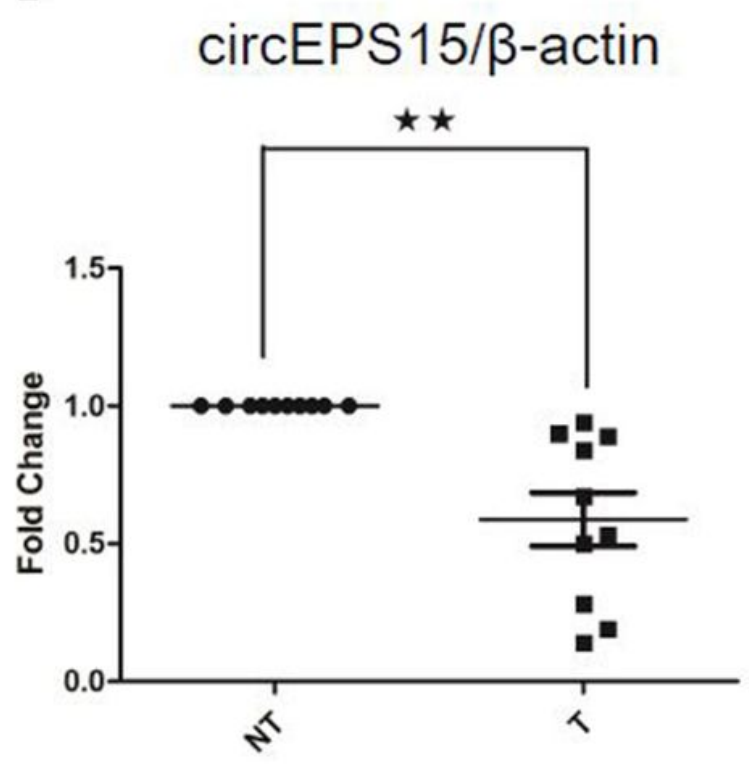

C
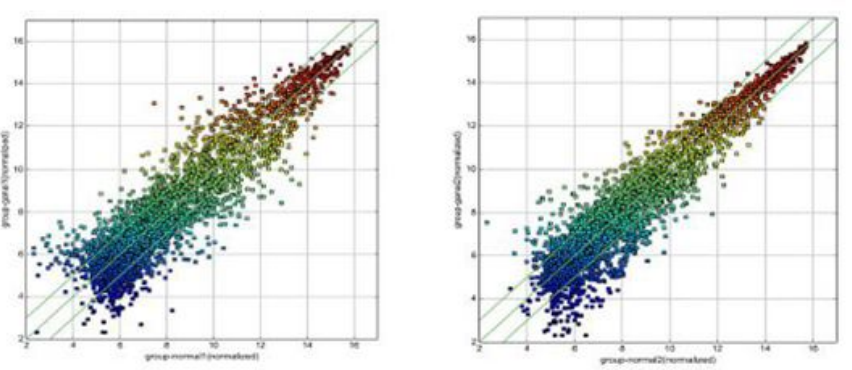

D
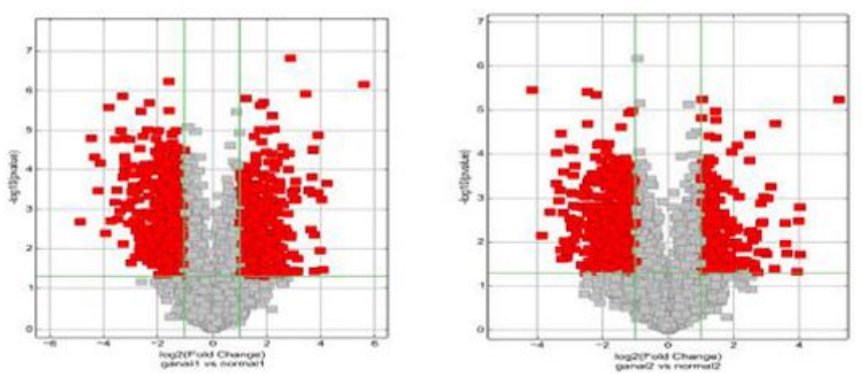

\section{circRNA-103595/ $/$-actin}

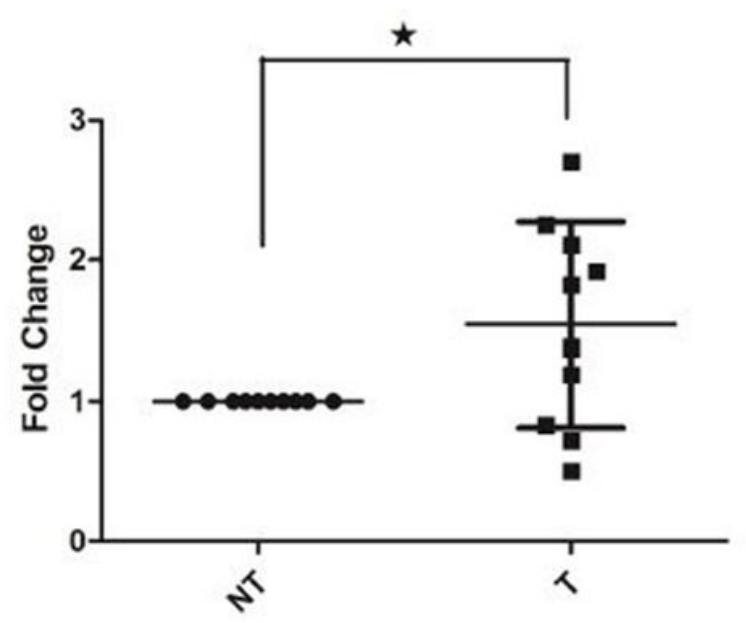

\section{Figure 1}

Differential expression of circRNAs between human hepatoma and adjacent tissues Microarray analysis was conducted to compare circRNA expression differences between normal and hepatoma human cells. a) Venn analysis of commonly upregulated and downregulated circRNAs indicating that some circRNAs have their specific expression characteristics in tumor heterogeneity. b) Heap map of differentially expressed circRNAs between three patients of normal and tumor tissues (significant fold change $>4$ ).c) Scatter plots depicting the raw variations in the expression profile between the two tissues types. d) A 
volcano plot depicting fold change and $p$-value between hepatoma and adjacent tissues. The horizontal line represents $P=0.05$ and the red points represent the circRNAs that are statistically different between the two cell types investigated. The green lines in a and b represent a 1.5 -fold change in expression. e) Expression levels of circEPS15 and circRNA-103595 in 32 randomly selected paired hepatoma and adjacent tissue samples detected with junction primers $(P<0.01)$.

\section{Fig. 2 A}

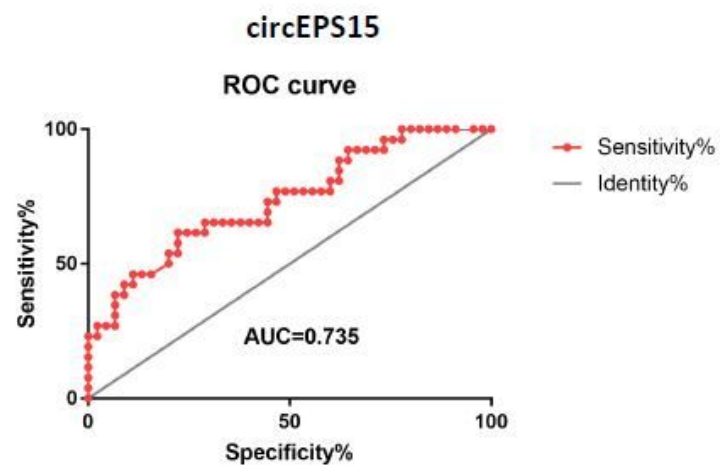

C

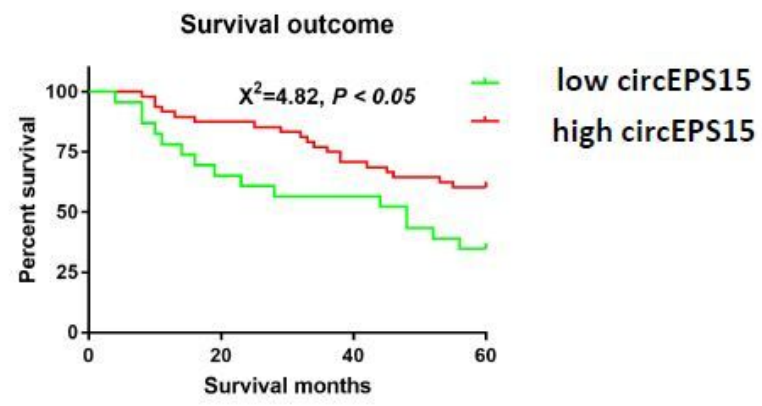

B $\quad$ circRNA-103595

ROC curve

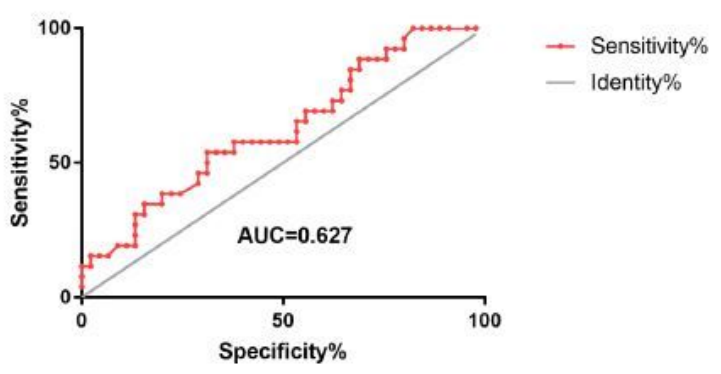

\section{Figure 2}

Profiling and characterization of circEPS15 as a circular RNA in human hepatoma and adjacent tissues a-

b) Combined with the prognosis results from these patients, an ROC curve was constructed, and AUC of 0.735 and 0.627 for circEPS15 and circRNA-103595, respectively, was obtained, indicating that the expression of circEPS15 has a better predictive value for prognosis in HCC patients. c) Kaplan-Meier's survival curves depict the correlation between circEPS15 and the overall survival of HCC patients. The patients were stratified into two groups according to a cut-off value of 0.8796 for circEPS15. Patients with low circEPS15 expression had a shorter overall survival compared to patients with high circEPS15 expression. There are 71 patients included in each experiment, and values indicate the average \pm SD of three independent experiments. The Student's two-tailed unpaired test and Log-rank test were used for statistical analysis of these data. 


\section{Fig.3}

A

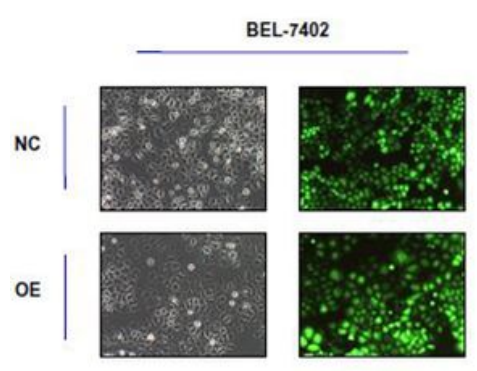

BEL-7402

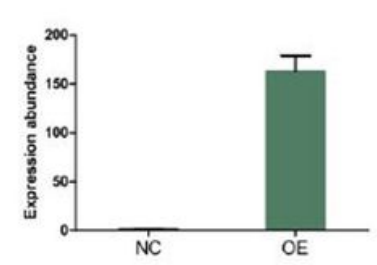

BEL-7404

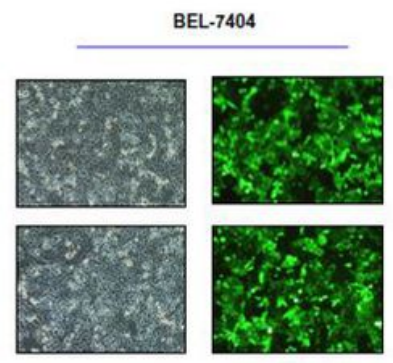

BEL.7404

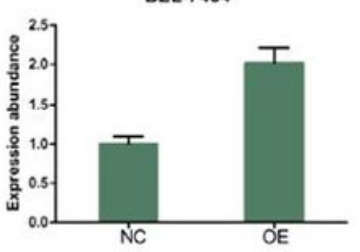

B
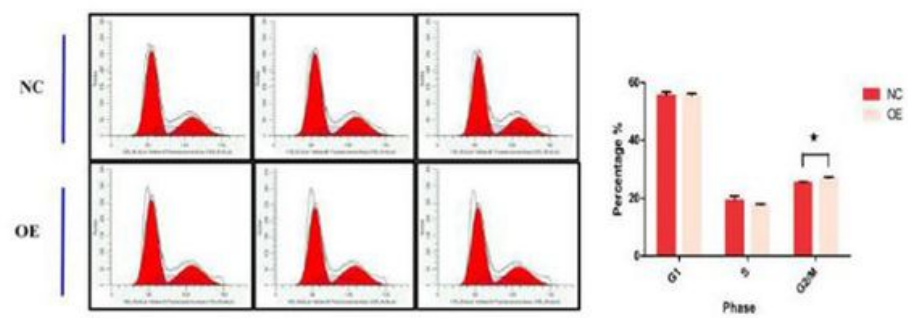

C
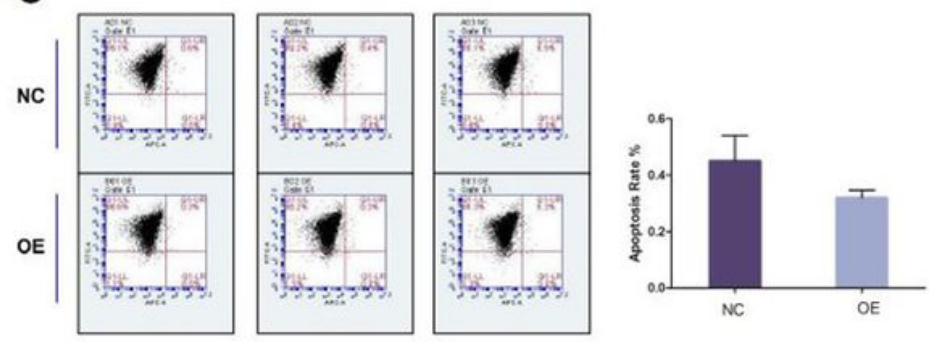

D
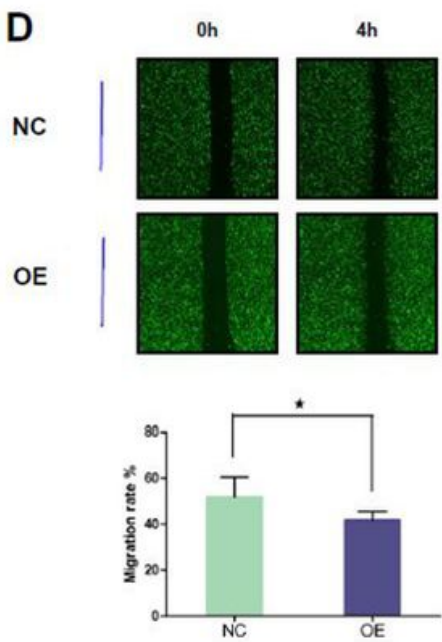

$4 h$
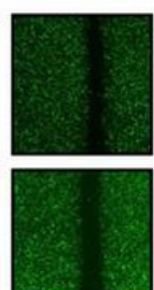


F
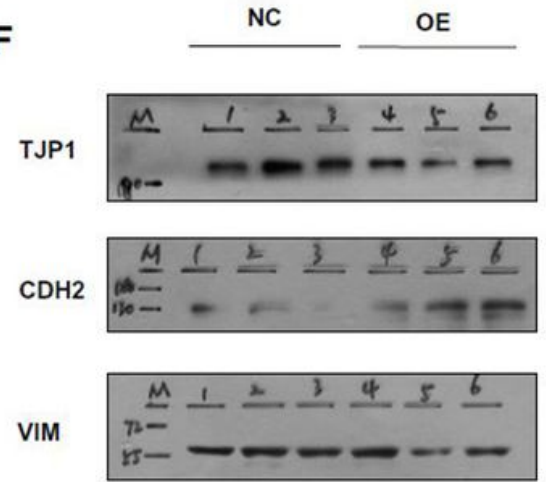

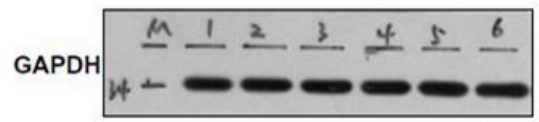

\section{Figure 3}

circEPS15 suppressed HCC cell proliferation, migration and invasion a) Real-time PCR was used to detect circEPS15 expression in Bel-7402 and Bel-7404 cells transfected with circEPS15 overexpression adenovirus constructs or an empty vector control $(P<0.01)$. b-c) Flow cytometry was used to detect cell cycle and cell apoptosis progression in transfected Bel-7402 cells $(P<0.01)$. d) Migration of cells overexpressing circEPS15 or the negative control was measured in a wounding assay at $0 \mathrm{~h}$ and $48 \mathrm{~h}$. The bar chart illustrates the relative migration distance of cells into the wounded region. The data are 
mean \pm SEM of three independent experiments $(P<0.01$, compared to $0 \mathrm{~h})$. e) A trans-well assay was used to evaluate the invasion ability of cells overexpressing circEPS15 or the empty vector control) after $48 \mathrm{~h}$. The bar chart illustrates the relative percentage of migrating cells. The data are mean \pm SEM of three independent experiments $(P<0.01$, compared to the mock control). $f)$ Representative western blotting images show that TJP1 expression in Bel-7402 cells decreased and $\mathrm{CDH} 2$ expression increased in circEPS15 overexpression cells compared with those transfected with the empty vector. However, there was no significant change in VIM expression.

\section{Fig.4}

hsa-miR-24-3p vs circEPS15



\section{hsa-miR-138-5p vs circEPS15}

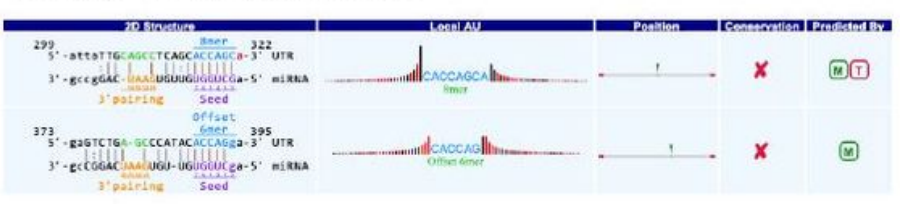

hsa-miR-145-3p vs circEPS15

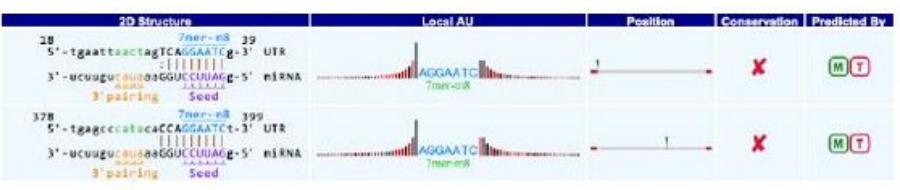

\section{hsa-miR-620 vs circEPS15}

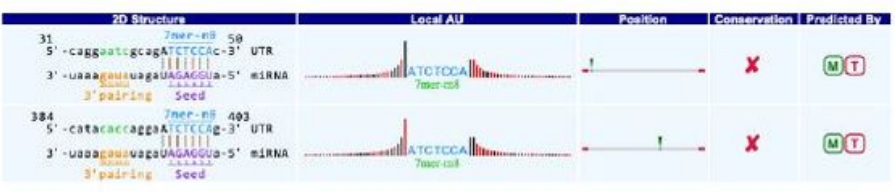

hsa-miR-875-3p vs circEPS15

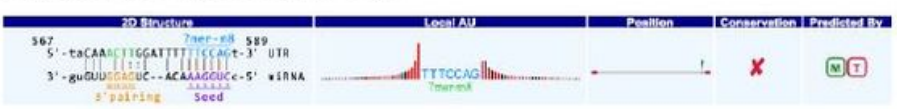

Figure 4

Detailed annotation of circRNA-miRNA interactions Predicted interaction sites of MicroRNA Respones Elements (MREs) circEPS15. M: be predicted by miRanda; T: be predicted by TargetScan. 
Fig.5

A

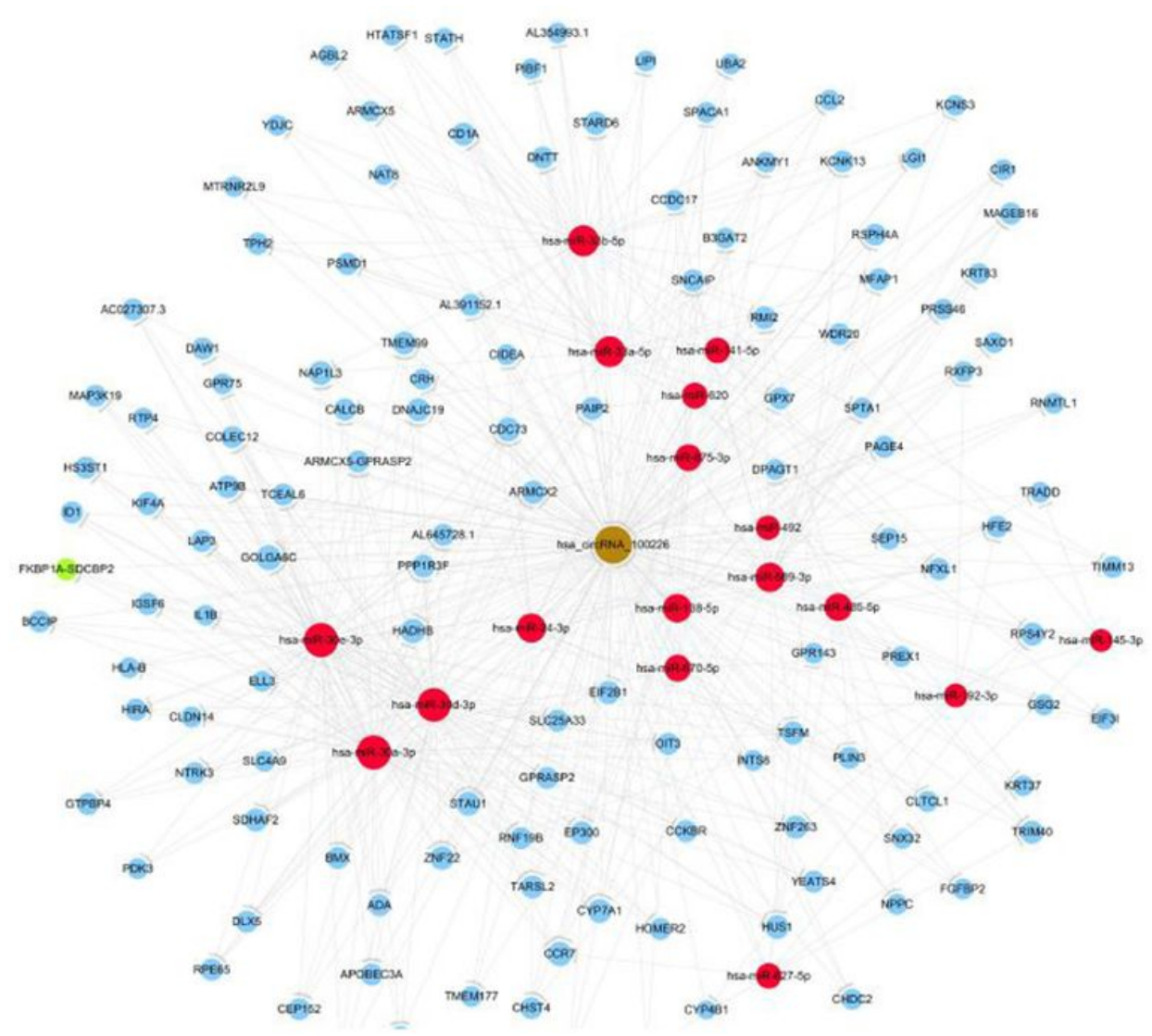

B

C
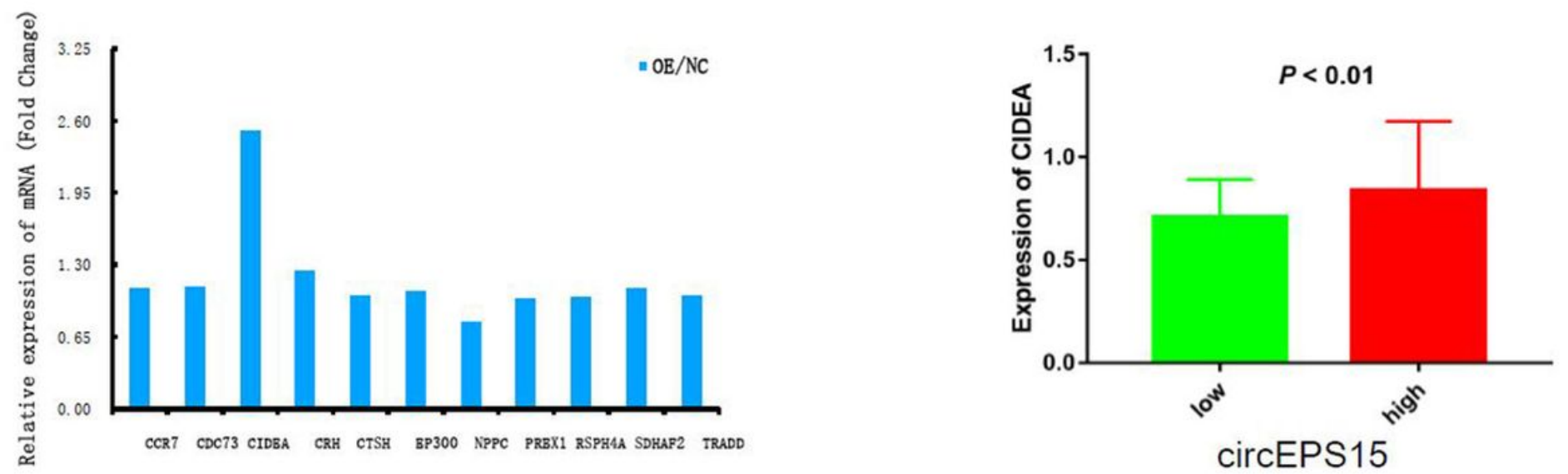

\section{Figure 5}

Predicted circRNA-miRNA-mRNA networks An expression network between circEPS15, miRNA and mRNA was constructed using a network diagram. a) The red and light-blue colors represent miRNA and mRNA, respectively, and brown represents circEPS15. Edges with T-shape arrows represent directed relationships, while edges without arrows represent undirected relationships. b) Eleven target genes were tested in Bel7402 cell transfected with a circEPS15 overexpression adenovirus construct (OE) or empty vector control (NC) by qRT-PCR. c) CIDEA mRNA and circEPS15 expression was detected in human hepatoma and 
adjacent tissues. Correlation analysis showed that there was a positive correlation between the expression of CIDEA and circEPS15.

A

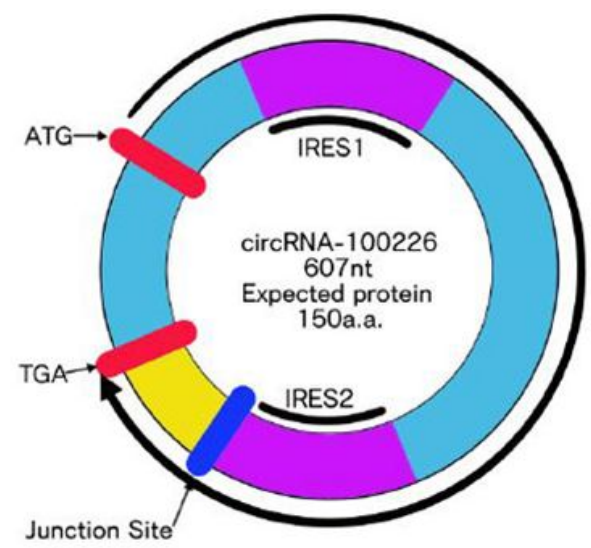

hsa_circ_100226 Mature Sequence

ATCTCTTCTCTGAAAGCTGAATTAACTAGTCAGGAATCGCAGATCTCCACTTACGAAGAA GAATTGGCAAAAGCTAGAGAAGAGCTGAGCCGTCTACAGCAAGAAACAGCAGAATTGG AGGAGAGTGTAGAGTCAGGGAAGGCTCAGTTGGAACCTCTTCAGCAGCACCTACAAGAT TCACAACAGGAAATTAGTTCAATGCAAATGAAACTGATGGAAATGAAAGATTTGGAAAA TCATAATAGTCAGTTAAATTGGTGCAGTAGCCCACACAGCATTCTTGTAAACGGAGCTAC AGATTATTGCAGCCTCAGCACCAGCAGCAGTGAAACAGCCAACCTTAATGAACATGTTG AAGGCCAGAGCAACCTAGAGTCTGAGCCCATACACCAGGAATCTCCAGCAAGAAGTAGT CCTGAACTACTGCCTTCTGGTGTGACTGATGAAAATGAGGTGACTACAGCTGTTACTGAA AAAGTTTGTTCTGAACTCGACAATAATAGACATTCAAAAGAGGAAGATCCATTTAATGTA GACTCAAGTTCGCTGACAGGTCCAGTTGCAGATACAAACTTGGATTTTTTCCAGTCTGAT CCTTTTGTTGGCA

B

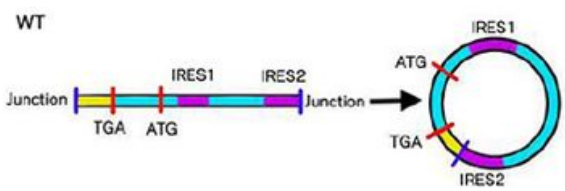

OE5 (WT+FLAG)

FLAG: GACTACAAAGA GATGACGACAAG

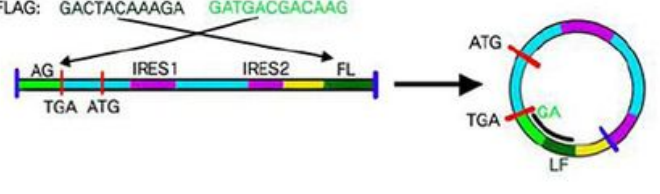

C

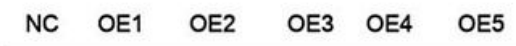

Flag

GAPDH
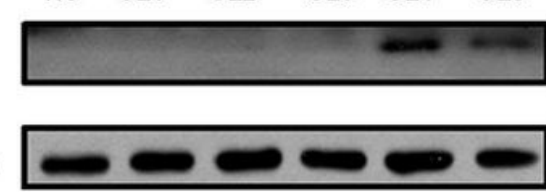

OE1

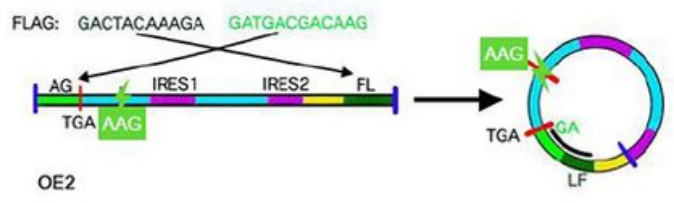

FLAG: GACTACAAAGA GATGACGACAAG

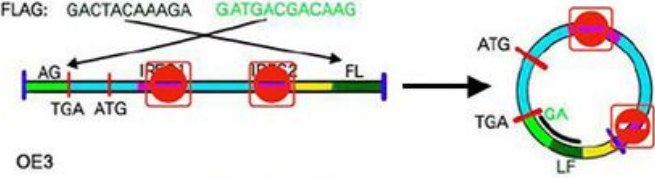

FLAG: GACTACAAAGA GATGACGACAAG

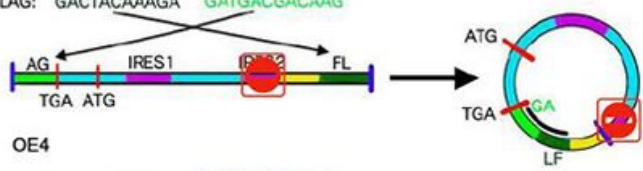

FLAG: GACTACAAAGA GATGACGACAAG

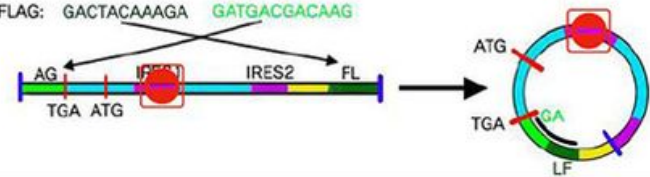

\section{Figure 6}

Evaluation of the protein coding ability of circEPS15 a) Upper panel: the putative open reading frame (ORF) in circEPS15. Note that the circEPS15 junction is inside the ORF. Lower panel: the sequences of the putative ORF are shown in black, internal ribosomal entrance site (IRES) sequences are shown in yellow, and start (ATG) and stop (TGA) codons are shown in red. b) WT: illustration showing how endogenous 
circEPS15 is formed; the circular junction is inside the ORF and forms the unique sequences (yellow). OE5: a FLAG tag was divided to both sides (light and dark green). Circularization of this vector formed the same circular RNA as the endogenous circEPS15, except with a FLAG tag added behind the ORF. OE1: the start codon was mutated from ATG to AAG to clarify the role of the start codon. OE2: IRES sequences (IRES1 and IRES2) in circEPS15 were truncated. OE3: IRES sequences (IRES2) in circEPS15 were truncated. OE4: IRES sequences (IRES1) in circEPS15 were truncated. Lower: a FLAG tag antibody was used to detect circEPS15 expression in 293T cells transfected with the vectors described above. 